\title{
METAL ENRICHMENT OF LY ALPHA CLOUDS AND THE INTERGALACTIC MEDIUM
}

\author{
I. MURAKAMI \\ National Institute for Fusion Science, Toki, 509-5292, Japan \\ AND \\ K. YAMASHITA \\ Information Processing Center, Chiba Univ., Chiba 263, Japan
}

We have considered the metal enrichment of the intergalactic medium (IGM) and Ly $\alpha$ clouds at high redshifts. The CIV absorption lines associated with the Ly $\alpha$ forest have been observed (e.g. Songaila \& Cowie 1996) and the metallicity is estimated as $\sim 0.01 Z_{\odot}$. Here, based on a galactic wind model, we have examined how the IGM is metal-enriched by the outflows driven by supernova explosions in primeval galaxies and minihalos.

In a spherical cloud model for a minihalo which experiences star formation, supernova explosions cause the expanding gas shell which propagates towards the collapsing envelope. The expanding velocity depends on the star formation timescale. The diffuse hot gas behind the shell is polluted by metal and expands with the shell.

The propagation of metallic gas into the IGM, where diffuse void regions and dense gas walls exist, has been examined in a 3D toy model of grid geometry. The outflow towards the dense wall is prevented as expected. Metallic gas is accumulated mostly at the dense shell, but the metallicity is nearly uniform at the shell and in the hot cavity behind the shell.

The 3D hydrodynamical simulation for the IGM in the CDM model is performed and we examine the metallicity distribution of the IGM. At $z=3$ only $10 \%$ of the IGM has metallicity $Z>10^{-2} Z_{\odot}$, and this volume fraction increases up to $30 \%$ at $z=0$. The void region is almost metal free and the metallic gas traces the filamentary structures of the matter distribution.

\section{References}

Songaila, A. \& Cowie, L.L. 1996, AJ, 112, 335 